\title{
L'ÉTAT GUATÉMALTÈQUE ET LES POPULATIONS MAYAS : STRATÉGIES D'IDENTIFICATIONS ETHNIQUES NÉGOCIÉES CHEZ LES CHUJ (1821-2011)
}

Carine Chavarochette

Presses de Sciences Po | «Critique internationale »

2013/3 N 60 | pages 133 à 150

ISSN 1290-7839

ISBN 9782724633139

Article disponible en ligne à l'adresse :

https://www.cairn.info/revue-critique-internationale-2013-3-page-133.htm

Distribution électronique Cairn.info pour Presses de Sciences Po.

(C) Presses de Sciences Po. Tous droits réservés pour tous pays.

La reproduction ou représentation de cet article, notamment par photocopie, n'est autorisée que dans les limites des conditions générales d'utilisation du site ou, le cas échéant, des conditions générales de la licence souscrite par votre établissement. Toute autre reproduction ou représentation, en tout ou partie, sous quelque forme et de quelque manière que ce soit, est interdite sauf accord préalable et écrit de l'éditeur, en dehors des cas prévus par la législation en vigueur en France. Il est précisé que son stockage dans une base de données est également interdit. 


\title{
L'État guatémaltèque et les populations
} mayas : stratégies d'identifications ethniques négociées chez les Chuj (1821-2011)

\author{
par Carine Chavarochette
}

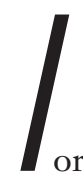

ors du recensement de 2005, le Guatemala comptait 14655189 habitants, dont $40 \%$ à $55 \%{ }^{1}$ se déclaraient d'ascendance « maya $»^{2}$. De l'époque coloniale aux années 1950, ce pays de l'isthme a orienté son économie vers l'agriculture exportatrice, principalement la mono-exportation de café et de bananes. Contrairement aux pays andins, il n'a pas fondé sa richesse sur l'extraction minière alors même que son sous-sol est riche en minerais divers (nickel, zinc, cuivre, plomb, et dans une moindre mesure or et argent). De plus, l'urbanisation et l'industrialisation, beaucoup plus tardives que dans le reste du continent, sont restées limitées. La mono-exportation agricole repose en effet sur la possibilité de disposer de grandes étendues de

1. Cette amplitude est due à la diversité des critères et des catégorisations utilisés par l'État lors des recensements (locuteur d'une langue indienne, auto-ascription, etc.).

2. Nous utilisons les guillemets pour souligner le caractère plurivoque de ce terme et sa construction historique. Santiago Bastos, Brett Roddy, El movimiento maya en la década después de la paz (1997-2007), Guatemala, FyG Editores, 2010 ; Agnès Bergeret, « Anthropologie et controverse identitaire au Guatemala », Fournal des anthropologues, 110-111, 2007, p. 161-183. 
terres, peu chères et de bonne qualité, ainsi que sur une main-d'œuvre bon marché et corvéable à merci, via la pratique de l'endettement systématique qui attache les paysans à la terre qu'ils travaillent pour le compte d'un grand propriétaire. Or c'est principalement sur une partie de la population « maya » que ce système économique a longtemps reposé et repose encore aujourd'hui dans une moindre mesure.

Depuis le $1^{\text {er }}$ janvier 1994, date de l'entrée en vigueur de l'Accord de libreéchange entre le Canada, les États-Unis et le Mexique (ALENA), le Guatemala est en outre devenu l'espace frontière entre l'Amérique du Nord et l'Amérique latine. Après une succession de régimes autoritaires, une révolution avortée et une guerre civile de trente-six ans, la démocratisation s'y révèle être un processus laborieux. Contrôlé par une élite métisse et/ou «blanche », l'État a en effet promu pendant des décennies une ségrégation raciale limitant la participation politique des populations indiennes.

À partir de l'exemple de la région du Nord-Ouest du Guatemala, plus précisément du département de Huehuetenango ${ }^{3}$, région frontalière avec le Mexique, ancienne zone de guerre et aujourd'hui zone de migrations internationales, nous questionnerons les expressions de l'ethnicité entendue ici comme un processus social dynamique et non comme l'expression d'une tradition culturelle archaïque 4 . En effet, les acteurs politiques (non seulement les partis, mais aussi les groupes de guérilla et l'armée) ainsi que les associations civiles (« mayas » et non-« mayas ») ne cessent de redéfinir leurs stratégies d'identifications et leur place au sein de la société guatémaltèque. Nous reviendrons tout d'abord sur la relation entre l'État et les Indiens chuj depuis la fin du XIX è siècle jusqu'à la période démocratique de 1944-1954. Nous analyserons ensuite les politiques d'assimilation et la violence de l'État à l'égard des populations indiennes et notamment chuj. Enfin, nous reviendrons sur l'apparition et l'évolution du terme « maya » ainsi que sur la mobilisation et l'instrumentalisation de l'ethnicité par les acteurs civils, qui cherchent à résoudre les problèmes inhérents à leur statut dans la société et à participer aux décisions politiques nationales, voire continentales.

\section{L'État guatémaltèque et les populations indiennes de la zone frontière}

Au Guatemala, les combats pour l'indépendance furent moins nombreux que dans d'autres régions de l'empire espagnol (Mexique, Venezuela, HautPérou, par exemple). Sous la pression du Mexique qui menaçait de l'envahir, le Guatemala déclara son indépendance en 1821 mais fut pourtant rattaché de

3. Département créé officiellement en 1866.

4. Fredrik Barth «Les groupes ethniques et leurs frontières », dans Philippe Poutignat, Jocelyne StreiffFénart, Théories de l'ethnicité, Paris, PUF, 1995 (1969). 
1822 à 1823, comme les autres provinces de l'Amérique centrale, à l'Empire mexicain d'Iturbide. Par la suite, Guatemala, Salvador, Honduras, Nicaragua et Costa Rica formèrent la Fédération d'Amérique centrale, avant de devenir cinq États indépendants à partir de 1839.

Dès lors, le Guatemala et le Mexique durent fixer leurs limites territoriales dans une zone qui, sous l'ancien régime, dépendait de l'Audience du Guatemala et non de la vice-royauté de la Nouvelle Espagne. De 1821 à 1882, aucun tracé ni aucun traité ne fut adopté et la frontière internationale entre ces deux pays resta donc inexistante sur le plan juridique. Pourtant, les enjeux politiques de cette délimitation étaient importants : au cours de la seconde moitié du XIX $\mathrm{X}^{\mathrm{e}}$ siècle, les dirigeants guatémaltèques, qu'ils soient libéraux ou conservateurs, justifièrent leurs revendications territoriales tantôt en utilisant, tantôt en rejetant les populations indiennes $c h u j^{5}$ vivant dans cette zone.

Les archives mexicaines ${ }^{6}$ relatent ainsi que le 25 octobre 1873, la finca Gracias a Dios, propriété de Mariano Jesus Guillen, fut envahie par des troupes armées provenant du Guatemala. À cette époque, ce domaine était, semblet-il, située en terre mexicaine et non en terre guatémaltèque, comme ce fut le cas par la suite. Le beau-frère du propriétaire vint déposer plainte auprès des autorités de la Gobernación de Chiapas accusant « une force armée venant de la République du Guatemala et un groupe considérable d'Indiens chuj d'avoir pénétré dans le territoire de cette République [mexicaine] jusqu'aux environs de sa finca (...) et [d'être entrés] à l'intérieur de son terrain. (...) Étant venus pour parcourir la ligne [frontière], ils étaient parvenus jusqu'à un terrain situé en face de la finca (...) et une fois arrivés là, les indigènes avaient manifesté l'intention de brûler les baraquements de ladite finca et, pour ce faire, avaient apporté des branches de pin enflammées $\gg^{7}$. Ce finquero précise que des Indiens $c h u j$ avaient déjà envahi des terres mexicaines quelques jours auparavant, détruisant les marques faites par les autorités pour délimiter la frontière. Les Chuj peuplaient en effet les terres proches de la future frontière, ainsi que les régions de San Sebastian Coatan et San Mateo Ixtatan situées à plus de $80 \mathrm{~km}$ de celle-ci. S'ils étaient encore majoritaires dans la région, l'arrivée de migrants ladinos $^{8}$ (originaires de la capitale départementale ou du Chiapas voisin) et de migrants européens à la recherche de grandes étendues de terres commençait déjà à bouleverser en profondeur les rapports sociaux, faisant de la possession des terres un enjeu capital.

5. La langue chuj est une langue maya, rattachée à la branche q'anjob'al majeure. Les Chuj occupaient à l'époque coloniale le Nord-Ouest des montagnes des Cuchumatanes au Guatemala et plus particulièrement le site de San Mateo Ixtatan ; ils cultivaient également des terres situées près de la limite internationale actuelle et même au-delà, au Chiapas voisin.

6. Secretaría de Relaciones Exteriores (SRE), Mexico, 1873, n 30, Terrenos nacionales, p. 222.

7. Ibid., p. 225.

8. Ce terme désigne au Guatemala un Indien s'exprimant seulement en espagnol ou un non-Indien. 
Les travaux des géomètres, tant mexicains que guatémaltèques, chargés d'établir la limite entre le Mexique et le Guatemala suscitaient donc à l'évidence le mécontentement des populations locales, en raison de la pression exercée sur le marché foncier. Les archives prennent amplement en compte le témoignage des ladinos qui revendiquent la propriété exclusive des terres agricoles et ne mentionnent que partiellement la décision des Chuj de brûler des baraquements et de détruire les traces des mesures provisoires prises par les géomètres. On peut toutefois affirmer que les Chuj réagirent avec force aux décisions politiques concernant la délimitation des territoires mexicain et guatémaltèque, et à la division des terres qu'on voulait par là même leur imposer.

Ces documents mexicains signalent par ailleurs que des troupes armées guatémaltèques se trouvaient là au moment des faits (sans préciser leur rôle exact) et que les Chuj modifiaient les tracés de délimitation de la frontière, sans doute effectués par les autorités mexicaines : « Le capitaine Daniel Morales de la République du Guatemala est passé par Nenton avec ses troupes ; il est venu accompagné d'Indiens chuj pour examiner la ligne ou frontière [passant par] Gracias a Dios $»^{9}$. Pour les autorités guatémaltèques, les Chuj étaient en effet les meilleurs guides de la région parce qu'ils connaissaient les terres et leurs bornages. Elles se servaient donc de leurs connaissances pour revendiquer des territoires face aux Mexicains et légitimer leurs prétentions souveraines. Par la suite, les conflits de terres et de délimitations des parcelles s'accentuèrent lors du tracé de la limite internationale : « Manuel Reyes, de la République du Guatemala, dit qu'il avait constaté l'arrivée de cinquante hommes armés de Huehuetenango, dont l'objectif était de réunir les villages et les hameaux frontaliers de cette République et d'envahir la nôtre pour voler et incendier les fincas frontalières de cette République $\gg^{10}$. La frontière agricole, dans le sens d'occupation et de valorisation des terres par l'agriculture extensive et l'élevage bovin, est donc repoussée et les Guatémaltèques comme les Mexicains tentent d'étendre au maximum leurs possessions territoriales avant que ne soit établi le tracé définitif.

Dès que la frontière internationale est fixée, en 1882, les Chuj se heurtent à une politique gouvernementale qui les prive peu à peu de l'usufruit des terres de la zone, mais la Révolution mexicaine de 1915 et ses effets directs (trafics d'armes et de bétail le long de la frontière, arrivée d'exilés mexicains) obligent le gouvernement guatémaltèque à changer radicalement de politique. Les archives datées de cette année signalent ainsi que, grâce aux travaux de nouveaux géomètres guatémaltèques, les Chuj sont parvenus à faire valoir 
d'anciens titres de propriété et à recouvrer une bonne partie de leurs terres ${ }^{11}$. Ainsi l'État guatémaltèque naissant s'est-il appuyé sur les populations chuj de la zone frontalière pour asseoir sa souveraineté. Ensuite, il a refusé de reconnaître leurs droits à exploiter les terres de cette zone dès lors que celles-ci ont fait l'objet d'une politique de colonisation et de développement de l'agro-exportation.

De 1839 à 1871, le pays fut dirigé par des gouvernements conservateurs qui maintenaient les populations indiennes dans des rapports sociaux et ethniques fondés sur la discrimination. Pour développer la culture mono-exportatrice de café (80 \% des exportations nationales en 1880), ils commencèrent à exproprier les communautés et à mettre les terres en vente. Ce mouvement s'accéléra avec l'arrivée des libéraux au pouvoir en 1871 : Justo Rufino Barrios (1873-1885) incarna cette politique de développement de la propriété privée, corollaire d'une économie capitaliste de mono-exportation agricole financée par des capitaux étrangers. Dans un contexte où la propriété collective apparaissait comme un legs du passé, la modernisation du pays passait par la suppression d'un régime foncier spécifique pour les populations indiennes. Les terres indigènes parcellisées et réduites firent alors l'objet d'un accaparement et d'une spoliation systématiques. Grâce au procédé juridique de « dénonciation des terres en friche $\gg$ (denuncios de tierras baldias) dans de nombreuses régions du pays, la population ladina en pleine expansion et les nouveaux immigrants européens purent acheter à bas prix des milliers d'hectares de terres publiques ainsi que des terres déclarées officiellement en friche, mais le plus souvent exploitées par les populations indiennes (cultures vivrières, cueillettes, pêche, etc. $)^{12}$. Entre 1871 et 1883, le gouvernement Barrios revendit à des planteurs de café plusieurs centaines de milliers d'hectares de terres publiques jusqu'alors exploitées par des paysans indiens des hautes terres qui y pratiquaient une agriculture de subsistance. Entre 1896 et 1918, pour les sept départements où la concentration de population indienne était la plus forte, plus de $45 \%$ des lots de terres concédés par le gouvernement à des particuliers appartenaient à des personnes d'origine espagnole ou européenne, essentiellement des Allemands. Attirés par le récent boom du café, ces nonIndiens s'installèrent dans les départements des hautes terres (altiplano) où ils firent fortune en tant que commerçants, vendeurs de liqueurs et pourvoyeurs de main-d'œuvre aux planteurs.

À cette époque, la région était perçue comme un réservoir de terres disponibles, un front pionnier, où ne parvenaient pas toutes les décisions politiques

11. Archivo General de CentroAmérica (AGCA), Guatemala Ciudad, Tierras, Paquete 33, exp.20 et Jefatura politica de Huehuetenango, leg.47785, 1943.

12. Sur ce procédé juridique, voir Carine Chavarochette, Frontières et identités en terres mayas $\left(X I X^{e}-X X I^{e}\right.$ siècle), Paris, L'Harmattan, 2011, chap. 3. 
prises à la capitale. La limite internationale était plus une frontier (frontière mobile et ouverte) qu'un border (frontière fermée qui divise). Par ailleurs, cette zone restée en marge de la colonisation avait été longtemps dépeuplée et les villages indiens y étaient disséminés. Les autorités du XIX ${ }^{\mathrm{e}}$ siècle estimèrent que les populations chuj qui vivaient là n'avaient pas l'utilité de ces terres, et qu'il était donc préférable de les offrir à des migrants ladinos ou allemands qui les exploiteraient et permettraient au Guatemala de développer une économie d'agro-exportation. Les bornes furent alors déplacées ; les Chuj vivant à l'extérieur de San Mateo Ixtatan et de San Sebastian Coatan perdirent la majeure partie de leurs terres, donc leurs moyens de subsistance, et durent souvent travailler dans les nouvelles exploitations d'élevage et de café pour le compte des nouveaux arrivants.

Cette inféodation des Indiens aux nouvelles exigences économiques fut également marquée par des méthodes d'intégration forcée dans le marché du travail. Sous le gouvernement Barrios en 1877, les travailleurs ruraux étaient obligés, sous peine d'être enrôlés de force, de présenter un document sur lequel figuraient les obligations de leur contrat de travail. Les autorités politiques régionales avaient en effet le pouvoir de recruter par la force un certain nombre de personnes au sein de chaque communauté des hautes terres, pour les contraindre à travailler dans les nouvelles plantations de café (nationales et étrangères) de la côte Pacifique.

Le développement de cette monoculture du café eut également des conséquences culturelles importantes sur la place de l'Indien dans la définition de l'avenir du pays. Ce moment coïncide en effet avec l'émergence au sein de l'élite politique et sociale guatémaltèque d'une idéologie qui considère que les populations indigènes et leurs cultures constituent une entrave au progrès économique et social du nouvel État. Dès lors, toute manifestation de la culture indigène - le port d'un costume traditionnel, l'usage d'une langue maya et surtout la propriété collective des terres - apparaît comme une pratique archaïque tandis que l'entrée dans la modernité est synonyme d'assimilation, voire de suppression de la différence culturelle ${ }^{13}$. Cette conjonction des régimes autoritaires, de l'exploitation des populations indiennes et de la mono-exportation du café, qui se traduisait par la mainmise des grands propriétaires et des compagnies étrangères comme la United Fruit Company sur le pouvoir politique et l'économie du pays, atteint son sommet sous la présidence autoritaire de Manuel Estrada Cabrera (1898-1920), mais elle prend fin avec la chute du général Jorge Ubico en octobre 1944. La révolution

13. Ces politiques d'assimilation et de démembrement des terres indiennes collectives existaient également de l'autre côté de la frontière. Dès 1824, le gouvernement mexicain encouragea la propriété privée au profit de l'agro-exportation, notamment au Chiapas, et différentes lois comme la loi Lerdo de 1856 s'inscrivirent dans cette volonté étatique d'assimiler l'Indien à la nouvelle nation mexicaine. 
qui prétend mettre un terme au « règne de l'oligarchie » ouvre le pays à des élections démocratiques. Sous les présidences de Juan José Arevalo (1945-1951) et de Jacobo Árbenz Guzman (1951-1954), les Guatémaltèques acquièrent de nouvelles libertés politiques.

\section{Entre assimilation et revendications}

Les gouvernements conservateurs et libéraux issus de l'Indépendance mais aussi l'exercice oligarchique du pouvoir par une élite blanche ont maintenu des pratiques racistes et autoritaires vis-à-vis des Indiens. En effet, depuis la fin du XIX ${ }^{\mathrm{e}}$ siècle, l'idéologie politique et sociale dominante était celle d'un « nationalisme exclusif $»^{14}$, c'est-à-dire excluant les populations indiennes de la conception de la nation et du corps politique guatémaltèque. L'éducation bilingue n'étant pas encore mise en place et le droit de vote étant refusé à tous ceux qui ne parlaient pas l'espagnol, les populations indiennes se voyaient exclues de facto de l'exercice de la citoyenneté. Au-delà de la question linguistique, l'organisation même de la vie politique les excluait de nombreux processus électoraux. Certes, la Révolution de 1944 entreprit de réformer en profondeur la société en luttant notamment contre la pauvreté et en reconnaissant la « discrimination historique » dont les populations indiennes avaient été victimes. Mais le nouvel État chercha surtout à améliorer sensiblement les conditions socioéconomiques de la classe moyenne et de la population indigène en les intégrant davantage dans les politiques nationales. C'est donc une conception assimilationniste qui fut à l'œuvre, les Indiens étant perçus comme des citoyens dont il était nécessaire d'améliorer le niveau de vie. Le gouvernement mit en application un Code du travail et lança d'importants programmes d'alphabétisation dans les zones rurales. Le nouveau contexte politique engendré par la Révolution de 1944 entraîna donc une reformulation à la fois de la définition de la société guatémaltèque et de l'identification indienne par une élite nationale composée d'intellectuels et de responsables politiques. L'Institut national indigéniste (INI) fut créé en $1945^{15}$ afin de mettre au point les méthodes les plus appropriées pour « intégrer l'Indien au sein de la culture nationale $\gg^{16}$. Grâce à des enquêtes de terrain menées dans des dizaines de villages indiens, l'INI répertoria les coutumes et les régimes fonciers pouvant varier d'un village à un autre. Ses actions ainsi que les politiques sociales engagées par l'État furent toutefois limitées dans la zone frontalière qui demeura une région enclavée.

14. Pour reprendre l'expression de Shelton H. Davis, « Mouvement maya et culture nationale au Guatemala », Fournal de la société des américanistes, 90 (2), 2004 (http://jsa.revues.org/1724).

15. L'INI fut également créé au Mexique et un bureau fut ouvert en 1948 au Chiapas.

16. S. H. Davis, « Mouvement maya et culture nationale au Guatemala », art. cité, p. 145. 
La Constitution de 1945 déclara hors-la-loi toutes les formes de discrimination et condamna l'exploitation de la main-d'œuvre indigène (Art. 67). Parallèlement, elle reconnut que les arts populaires étaient des éléments essentiels de la culture nationale (Art. 87), sans toutefois mettre en avant leur spécificité ethnique. Ces dix années de transition démocratique ne s'inscrivaient donc pas encore dans la promotion d'un pluralisme ethnique et culturel au Guatemala. Durant cette même période des années 1950, de nombreux paysans, obligés par l'accroissement démographique et la parcellisation des terres qui en découla directement de migrer comme travailleurs saisonniers dans les plantations d'autres régions, devinrent des paysans sans terre et perdirent leur statut social. Parallèlement, des mouvements soutenus par les différentes organisations catholiques, de l'Action catholique à la Démocratie chrétienne, suscitèrent le développement d'une mobilisation politique dans les villages indiens ${ }^{17}$.

Ce court intermède de pouvoir civil et démocratique en pleine période de guerre froide prit fin avec le coup d'État du général Castillo Armas qui, soutenu par les États-Unis, prit le pouvoir en 1954. Durant les trente années suivantes, d'autres généraux se succédèrent au pouvoir par la fraude électorale et les coups de force, et limitèrent les libertés publiques, individuelles et collectives, des citoyens. En 1966, les guérillas marxistes - qui étaient au demeurant totalement indifférentes à la question indienne - furent décimées par l'armée guatémaltèque. Ce fut seulement au début des années 1970 que s'organisèrent de nouveaux mouvements marxistes-léninistes, comme La Organización del Pueblo en Armas (ORPA) et l'Ejercito Guerrillero de los Pobres (EGP), qui « découvr[ir]ent les Mayas $»^{18}$ et pensèrent qu'une révolution ne pouvait réussir sans tenir compte de la population indigène et de ses revendications. Toutefois, à cette date, ces mouvements étaient essentiellement composés de ladinos. De leur côté, les Indiens paupérisés s'organisèrent dans des syndicats comme le Comité d'union paysanne (CUC) pour revendiquer notamment leurs droits à la terre et réagir à la violence de l'État dictatorial. Face à la répression des guérillas par l'armée et à l'incapacité des mouvements sociaux - étudiants, syndicats ouvriers et paysans, partis d'opposition - à déstabiliser le pouvoir militaire, la violence sociale atteignit son paroxysme et fit basculer le pays dans la guerre civile.

À la fin des années 1970, alors que la violence d'État était de plus en plus forte, la population rurale indigène parvint à se mobiliser massivement pour faire entendre ses demandes sociales (éducation, santé), économiques (hausse des

17. Yvon Le Bot, La guerre en terre maya : communauté, violence et modernité au Guatemala, Paris, Karthala, 1992 ; S. H. Davis, « Mouvement maya et culture nationale au Guatemala », art. cité ; Thomas Melville, Marjorie Melville, Tierra y poder en Guatemala, San José Costa Rica, EUA, 1975.

18. Y. Le Bot, La guerre en terre maya : communauté, violence et modernité au Guatemala, op. cit., p. 109. 
salaires, terres), culturelles (éducation bilingue, respect des pratiques culturelles) et politiques (citoyenneté, sécurité). Les demandes en matière d'éducation, de santé et de participation politique en particulier furent encouragées et relayées par les syndicats, les partis politiques d'opposition et certaines congrégations religieuses. Bien qu'elle fût variable selon les régions et les groupes ethniques, voire selon les individus au sein d'une même communauté, cette mobilisation sociale et politique devint générale. En outre, à la même époque, une nouvelle génération d'intellectuels et de leaders « mayas » qui avaient eu accès à l'enseignement secondaire ou supérieur réaffirmèrent leurs identifications ethniques face à des politiques nationales qui tentaient de les assimiler et de gommer leurs particularités au sein de la société nationale. Ainsi, certaines organisations œuvrèrent pour une reconnaissance de la culture indienne ; d'autres, plus politisées, se rapprochèrent des partis politiques de gauche et/ ou des syndicats, sans toutefois qu'aucun mouvement unifié ne se forme ${ }^{19}$. L'exemple des villages $c h u j$ du Nord-Ouest du pays illustre l'effet de surenchère provoqué par la politique de l'État militaire et démontre que, dans une guerre menée contre un ennemi politique - les membres des guérillas -, ce sont les populations civiles, notamment « mayas », qui, comme les Chuj, sont les premières victimes.

L'enquête menée par l'Oficina de Derechos Humanos del Arzobispado de Guatemala en 1998 a démontré que l'initiative des massacres venait le plus souvent du haut commandement militaire qui galvanisait les troupes en leur faisant croire à l'existence d'un butin à récupérer dans les villages. En effet, selon cet organisme, « la majorité des massacres commis ont été le produit de processus qui ont impliqué une préparation psychologique et matérielle. Beaucoup de décisions relatives à la destruction des hameaux ont été minutieusement planifiées et leur préparation exigeait également de maintenir un état psychologique de tension, d'alerte permanente et de capacité de réaction immédiate de la part de la troupe $»^{20}$.

Quatre massacres furent ainsi recensés dans le municipe de Nenton (situé au Nord-Ouest du département de Huehuetenango). Le premier, perpétré dans le village chuj de Yalambojoch en janvier 1982, fit une vingtaine de victimes et poussa les populations ( $c h u j$ et non $c b u j$ ) de la zone à prendre la fuite. Lors du massacre du 17 juillet 1982, le plus tristement connu, les forces régulières, composées essentiellement de soldats indiens d'autres régions et pratiquant

19. S. Bastos, B. Roddy, El movimiento maya en la década después de la paz (1997-2007), op. cit..

20. ODHAG, Guatemala. Nunca más (versión resumida). Informe del Proyecto Interdiocesano Recuperación de la Memoria Histórica, Guatemala, ODHAG, 1998, tome II, p. 220. Sur le genocide, voir également Marta Casaus Arzu, «El Genocidio : la máxima expresión del racismo en Guatemala : una interpretación histórica y una reflexión », Nuevo Mundo Mundos Nuevos, 2009 (http://nuevomundo.revues.org/57067) et Guatemala: linaje y racismo (Guatemala: Lineage and Racism), Guatemala, F\&G Editores, 2007 ; Yvon Le Bot, La Grande Révolte indienne, Paris, Robert Laffont, 2009. 
d'autres langues, tuèrent le bétail, détruisirent les récoltes et exterminèrent l'ensemble des habitants du petit village de San Francisco : plus de 300 Chuj de tous âges furent tués ; seuls cinq survécurent. En faisant massacrer des populations indiennes par des Indiens originaires d'autres régions, l'armée guatémaltèque parvint à mettre en évidence la faiblesse des liens intercommunautaires, du fait de la logique même de la guerre civile et du climat de violence extrême qui la constituait ${ }^{21}$.

Au mois de juin 1982, l'armée avait proposé aux habitants de San Francisco son appui et une aide économique à condition qu'ils ne soutiennent plus la guérilla ; dans le cas contraire, les soldats menaçaient d'exercer des représailles. L'EGP, qui avait installé son quartier général dans le Nord du département de Huehuetenango depuis la fin des années 1970, rayonnait alors dans ce municipe. En 1981, il avait lancé une campagne « d'élimination du pouvoir local de l'ennemi » en assassinant des personnes proches de l'armée, et avait poursuivi sa lutte contre les militaires sous le gouvernement de Rios Montt (23 mars 1982-8 août 1983). Toutefois, l'arrivée de 3000 soldats dans la zone en juin 1982 lui fit perdre de nombreux combattants, et il dut installer son quartier général dans la région voisine de l'Ixcan.

Les Chuj du village de San Francisco semblaient entretenir de bons rapports avec le propriétaire des terres sur lesquelles ils vivaient et travaillaient, un colonel retraité. En 1955, ce dernier avait réussi à leur obtenir à Yulaurel des terres contiguës aux siennes et à la frontière, dont l'acte notarié définitif ne leur avait été octroyé que dans les années $1970^{22}$. Au début des années 1980, la guérilla patrouillait dans cette zone, et comme elle avait assassiné des administrateurs de grands domaines, toutes les populations du municipe étaient suspectées de la soutenir. L'anthropologue Ricardo Falla ${ }^{23}$ pense que la (possible) collaboration de la population avec la guérilla fut la cause immédiate de ce massacre ; en effet, du point de vue des militaires, le fait de ne pas vouloir prendre parti revenait à soutenir la guérilla. L'hypothèse d'une volonté étatique de contrôle de la frontière a été également avancée ${ }^{24}$. Les enquêtes de terrain que nous avons menées de 1995 à 2001 ont révélé que, pour de nombreux habitants du municipe, le massacre s'inscrivait dans le conflit agraire de la région, l'extermination des populations pouvant permettre à d'autres grands propriétaires terriens non indiens de s'approprier les terres frontalières de Yulaurel. Après ce massacre, les populations civiles

21. Des habitants de la finca Nubila entre Dos Rios furent également tués par l'armée et par des membres des PAC (Patrouilles d'autodéfense civile), en juin 1982. Un quatrième massacre eut lieu au hameau de Tzala, au Sud de Nenton.

22. Y. Le Bot, La guerre en terre maya : communauté, violence et modernité au Guatemala, op. cit., p. 236.

23. Cité par AVANCSO, « Donde esta el futuro ? Procesos de reintegración en communidades de retornados », Cuadernos de investigación, 8, 1992, p. 97.

24. Ibid., et Y. Le Bot, La guerre en terre maya : communauté, violence et modernité au Guatemala, op. cit.. 
du municipe commencèrent à partir pour aller se réfugier au Mexique. Ceux qui n'avaient pas pu ou pas voulu fuir furent regroupés par l'armée dans des villages modèles, tandis que la guérilla était coupée de ses bases sociales et condamnée à agir dans une région dont les habitants avaient été évacués. Cette « politique de la terre brûlée » permit à l'armée de contrôler la frontière et d'empêcher la guérilla d'utiliser le Chiapas comme base arrière.

Ainsi, dans les années 1980, les agressions, les massacres de civils, les déplacements internes forcés et l'importance du nombre de réfugiés (plus de 1,5 million entre 1980 et 1983) provoquèrent la rupture des liens générationnels. Après la période des plus forts massacres et de l'exil au Mexique, de nouvelles organisations guatémaltèques apparurent pour dénoncer ces atteintes aux droits de l'homme, tandis que d'autres associations comme l'Académie des langues mayas du Guatemala, créée en 1986, ou le Comité des organisations mayas du Guatemala redonnaient un élan à la mobilisation indienne en l'inscrivant à l'agenda des revendications culturelles.

\section{Une nouvelle forme d'identification : I'apparition et l'appropriation du terme « maya »}

En Amérique latine, durant les années 1970, des leaders indigènes et des associations civiles commencèrent à remettre en cause la politique indigéniste des États latino-américains qui cherchaient à assimiler ou à intégrer, socialement et culturellement, d'importantes populations indiennes ${ }^{25}$. Au Guatemala, des intellectuels d'origine indigène et des leaders d'organisations indiennes dénoncèrent en particulier la conception de la nation construite autour de la figure du ladino, affirmèrent les droits des Indiens sur les terres qu'ils cultivaient et revendiquèrent la reconnaissance par l'État et les autres acteurs sociaux de leurs pratiques culturelles. Or ces mouvements qui réclamaient la reconnaissance de la pluralité ethnique, culturelle et linguistique de la société guatémaltèque utilisaient de plus en plus couramment le terme générique de «maya », en référence au désignant linguistique des populations indiennes. Ce faisant, ils reprenaient, volontairement ou non, le vocabulaire des archéologues et des anthropologues nord-américains qui avaient travaillé dans la région à partir des années $1940^{26}$. Au cours des années 1980, au moment où d'autres États latino-américains s'engageaient dans la transformation des

25. Cécile Casen, « Le katarisme bolivien : émergence d'une contestation indienne de l'ordre social », Critique internationale, 57, octobre-décembre 2012, p. 23-36.

26. Sur l'utilisation de cette catégorie, voir notamment Donna Lee Van Cott, From Movements to Parties in Latin America, Cambridge, Cambridge University Press, 2005. Les groupes ethniques désormais identifiés comme « mayas » pratiquent plus de 21 langues différentes. Les deux autres groupes indigènes reconnus par l'État guatémaltèque - les xinca (initialement situés sur la côte Pacifique et au Salvador) et les garifunas (présents sur la côte caraïbe) - ne se rattachent pas aux « mayas ». 
politiques culturelles nationales en reconnaissant la diversité ethnique et le pluralisme culturel dans leurs constitutions, le Guatemala, lui, s'enfonçait dans la guerre civile. Ce n'est que dans les années 1990, avec la fin des dictatures et l'installation de gouvernements démocratiques ralliés à l'idée néolibérale de gouvernance - et donc de participation accrue des acteurs sociaux à la gestion de leurs territoires -, que de nouveaux espaces politiques s'ouvrirent aux revendications des peuples indigènes. Autrefois inscrite dans une problématique économique et sociale, la lutte pour le droit à la terre prit dès lors sa place, aux côtés des revendications strictement culturelles (reconnaissance des langues mayas, éducation bilingue), dans un répertoire nouveau, celui de l'autochtonie.

L'imposition dudit répertoire dans la plupart des pays latino-américains fut en partie le résultat de facteurs externes, tels que la Déclaration des droits des peuples indigènes des Nations unies du 13 septembre 2007 et la Convention 169 de l'OIT portant sur les peuples indigènes et tribaux, votée en 1991. Toutefois, les modalités internes d'un tel changement varièrent selon les situations nationales : dans des pays comme la Bolivie, le Brésil, la Colombie ou l'Équateur, il se produisit dans le cadre d'un processus démocratique, fait de revendications et de concessions permettant aux organisations indigènes de se faire entendre des parlementaires, voire d'intégrer à leur tour les structures de représentation. En revanche, dans d'autres régions comme le Guatemala ou le Chiapas mexicain, ce fut par des conflits armés que les droits des indigènes s'imposèrent à l'opinion publique et furent éventuellement reconnus dans le contexte des négociations de paix entre gouvernements et groupes insurgés. Dans le cas du Guatemala, la nécessité s'imposa, à la fin de la guerre civile, de reconnaître la pluralité des identifications ethniques au sein de la nation guatémaltèque. Selon Shelton Davis ${ }^{27}$ et Santiago Bastos ${ }^{28}$, le mouvement «maya », quoique diffus, devint de plus en plus présent sur la scène publique et joua un rôle important dans le processus de paix des années 1990. Les différents groupes qui le composaient réclamaient non seulement des améliorations sociales et politiques, mais aussi la reconnaissance de leurs particularismes. En 1996, au lendemain des accords de paix ${ }^{29}$, le gouvernement dirigé par Alvaro Arzu ne pouvait plus nier la situation d'extrême pauvreté dans laquelle vivait une grande partie de la population indienne, qui représentait presque la moitié de la population du pays. Les élites et les intellectuels non mayas reconnurent alors pour la première fois le caractère pluriethnique de la nation,

27. S. H. Davis, « Mouvement maya et culture nationale au Guatemala », art. cité, p. 137-166.

28. Santiago Bastos, « La movilización maya en Guatemala : exigiendo derechos y construyendo multiculturalidad en un contexto de postconflicto », Cabiers des Amériques latines, 60-61, 2010, p. 41-58.

29. Outre le nouvel ordre mondial issu de la chute du mur de Berlin qui poussait les États-Unis et la communauté internationale à mettre fin à la guerre civile, la mise en place de l'ALENA et le soulèvement du Chiapas en 1994 conditionnèrent la paix au Guatemala. 
et les populations « mayas » elles-mêmes osèrent revendiquer haut et fort leur droit d'appartenir à celle-ci en tant que citoyens à part entière, tout en conservant leurs particularités. Un consensus s'opéra autour de l'idée selon laquelle, d'une part, l'appartenance à une ethnie ne devait plus constituer un facteur de discrimination, d'autre part, la classe politique « blanche » ou ladina ne devait plus dominer le reste de la société. Dès lors, les Indiens devaient pouvoir participer à la vie politique du pays, notamment au niveau local, et améliorer leurs conditions de vie. Signe des temps, la Constitution de 1985 fut traduite dans les quatre langues les plus parlées après l'espagnol (quiché, mam, cakchiquel, kekchi) et des documents officiels furent traduits dans les vingt-trois langues indiennes et non indiennes reconnues.

Il ne s'agissait pas seulement d'une « réconciliation » nationale mais plutôt d'une véritable définition de la nation, par la prise de conscience et l'acceptation, au sein de celle-ci, des populations «mayas » et de leurs singularités. Dès lors, le Guatemala se devait de relever le défi de ne plus fonder son projet politique sur un « nationalisme exclusif » qui niait la réalité ethnique de sa population. Or, en mai 1999, le référendum ayant pour objet de modifier la Constitution de 1985 et de reconnaître officiellement le Guatemala comme pays multiethnique et multiculturel, en concédant aux Indiens des droits spécifiques comme celui de bénéficier de lois coutumières, se solda par un échec. En fait, ce projet effrayait certains milieux conservateurs de la population ladina qui virent dans les revendications « mayas » une menace portée à l'unité de l'État.

Pourtant, la plupart des mouvements « mayas » ne proposent pas de projet de sécession et de création d'un État maya indépendant du Guatemala, pas plus qu'ils n'envisagent de promouvoir un « nationalisme exclusif » inversé, qui rejetterait tous les non-Mayas. Le mouvement mené par Eduardo Zapata tend à rassembler sous le concept de « nation » toutes les composantes de la population : Indiens, métis, ladinos et criollos. Ce courant, qui fait primer la citoyenneté sur l'ethnicité, appelle de ses vœux une nation certes pluriethnique et pluriculturelle, mais avant tout guatémaltèque. Pour sa part, Rigoberto Quemé Chay $^{30}$ relie les problèmes de pauvreté et de marginalisation ou de violence à la nature même de l'État guatémaltèque : raciste et archaïque. Il propose la création d'un État national où les revendications et les demandes particulières des Mayas seraient prises en compte avant tout comme des demandes émanant de Guatémaltèques. Le mouvement féminin porté par Irma Alicia Velazquez Nimatuj milite également pour la refonte de la société guatémaltèque sur des bases non raciales. Enfin, Demetrio Cojti ${ }^{31}$ représente la tendance qui envisage l'existence d'une nation ethnique, un État maya, mais

30. Ancien maire de la seconde ville du pays, Quetzaltenango.

31. Ancien ministre adjoint de l'Éducation nationale sous la présidence de Alfonso Portillo (2000-2004). 
qui serait intégré au sein d'un État fédéral : il ne prône donc pas la sécession mais parle de « souverainisme autonomiste ». Un dernier groupe, celui des femmes de Kaqla, illustre la diversité du mouvement actuel en proposant une réflexion collective sur l'identité des femmes indiennes et sur les obstacles qu'elles rencontrent dans leur vie quotidienne, réflexion qui contredit l'idée d'une culture et d'une identité « maya » figée et immuable.

Tous ces mouvements, dont les leaders ont en commun d'avoir exercé des fonctions dans l'administration locale ou centrale, revendiquent le rôle que doit jouer la société civile pour modifier et réformer en profondeur l'État guatémaltèque : ils veulent que cette institution soit accessible au plus grand nombre de citoyens. Tous critiquent l'État guatémaltèque monoethnique et monoculturel, incapable de répondre aux nécessités premières de sa population (économie, santé, éducation, sécurité). Mais ces mouvements ne sont pas homogènes ; certains leaders s'ancrent dans la sphère locale tandis que d'autres agissent au niveau national ${ }^{32}$.

Les organisations « mayas » ont joué un rôle majeur en promouvant une nouvelle conception de la nation guatémaltèque fondée sur l'idée de multiethnicité, de pluriculturalité et de multilinguisme. En 2007, pour la première fois, une femme maya, Rigoberta Menchú Tum, se portait candidate à la tête du pays sous l'étiquette Winaq-E-G, tandis que le choix d'un ladino producteur de café comme vice-président était critiqué par des associations indiennes et des membres de l'opposition. Dépourvue de moyens financiers (pour faire campagne) autant que de programme politique défini, Rigoberta Menchú n'a pas atteint les $5 \%$ à l'élection présidentielle. Cela étant, sa candidature n'était pas celle d'un parti maya unifié. Et lors de l'élection de 2011, il n'y a pas eu davantage de «parti maya » proprement dit.

Néanmoins, depuis quelques années, les populations indiennes comme les Chuj utilisent le terme « maya » dans leurs revendications sociales et politiques, comme en témoigne l'exemple de leur refus des implantations de mines sur leurs terres. Depuis 2005 en effet, plus de 400 licences ont été octroyées par les gouvernements guatémaltèques successifs à des multinationales pour exploiter des mines à ciel ouvert. Le département de Huehuetenango a été particulièrement touché par cette exploitation qui accélère la détérioration de l'environnement et des terres parce que son sous-sol contient les principales réserves de minerais (zinc, plomb, or, argent) du pays. Les populations indiennes de la région se sont donc organisées en « Conseil des peuples mayas de l'Occident » pour faire valoir leurs droits sur les terres qu'elles occupent et dénoncer les concessions accordées par le gouvernement et les

32. Sur le mouvement maya post-conflit, voir S. Bastos, B. Roddy, El movimiento maya en la década después de la paz (1997-2007), op. cit.; et sur la revalorisation de l'identité maya, Karine Vanthuyne, « Guatemala. Des ONG œuvrant pour la "paix" », Fournal de la Société des Américanistes, 90 (1), 2004, p. 240-258. 
autorités municipales.

Des organisations de lutte pour l'autodétermination, pour la défense des territoires ou encore pour la création d'un département « libre de mines et de tout mégaprojet (barrages, exploitations de pétrole) » ont été créées. Plusieurs organisations civiles dénoncent en outre les pressions physiques exercées par les multinationales (par le biais des autorisations municipales ou gouvernementales) sur les paysans afin qu'ils acceptent de vendre leurs terres aux entreprises minières. Sur l'un des sites web consacrés à la sauvegarde de la « Terre Mère » et à la lutte contre les implantations de mines à ciel ouvert, les populations indiennes directement concernées estiment « qu'elles vivent une troisième colonisation, après celle de l'Espagne et (...) du conflit armé $»^{33}$. En effet, ces implantations ont de graves répercussions sur l'environnement : l'utilisation de produits chimiques dont le cyanure, l'extraction du minerai, la destruction de la roche, du sol, la consommation élevée d'eau et la contamination des rivières et des sources affectent directement les populations, notamment les Chuj, majoritaires dans la région.

Depuis 2006, ces associations qui se revendiquent comme étant « mayas » organisent des consultations communautaires qui leur permettent de faire entendre leur voix auprès du gouvernement national et/ou municipal. Ces mobilisations civiles s'appuient sur la Loi générale de décentralisation, la Loi du Conseil de développement urbain et rural, le Code municipal et la Convention 169 de l'OIT stipulant que « les peuples indigènes doivent être consultés librement, préalablement et informés sur les points capitaux de leurs territoires $\gg^{34}$. Or, de 2005 à 2010, 41 licences d'extraction minière ont été octroyées dans le département de Huehuetenango sans qu'aucune consultation des populations n'ait été réalisée par le gouvernement. Ces assemblées dénoncent également la corruption généralisée qui permet ces concessions : celle de l'État vis-à-vis des multinationales étrangères, celle des autorités locales qui concèdent l'exploitation sur des terres municipales, voire celle des leaders communautaires qui cachent les effets de l'extraction minière sur les terres adjacentes à celle de la mine. Ainsi, les populations chuj qui combattaient au XIX ${ }^{\mathrm{e}}$ siècle l'implantation de grandes exploitations d'élevage ou de monoculture tournées vers le marché international se dressent au XXI ${ }^{e}$ siècle contre l'exploitation minière internationale.

En 2010, sur les 28 municipes composant le département de Huehuetenango, 13 s'étaient déclarés « territoires libres de mines ». Dans le municipe de Nenton, en 2007, 19847 personnes ont voté unanimement contre l'implantation de mines à ciel ouvert. Toutefois, dans son édition départementale du

33. Propos relayés par le Réseau de solidarité internationale NISGUA Working for Justice in Guatemala (nisgua.org).

34. Convention 169 de l'OIT ratifiée par le Guatemala en 1996. 
$1^{\text {er }}$ mars 2012, le quotidien Prensa Libre déclare que le nouveau gouvernement d'Otto Perez Molina (élu en novembre 2011) a accordé 37 nouvelles licences d'exploitation dans le département, y compris dans le municipe de Nenton. Depuis, un bras de fer est engagé entre les autorités gouvernementales et les associations civiles, ces dernières s'opposant à l'ouverture des mines et militant pour la promotion de ces consultations communautaires comme droit à s'opposer et à donner leur avis sur l'implantation d'un mégaprojet.

Dans ce conflit deux conceptions des ressources naturelles s'opposent : celle des multinationales qui considèrent que les matières premières ${ }^{35}$ sont un bien marchand comme un autre et celle des associations « mayas » (pouvant également regrouper des populations ladinas) qui considèrent que leur survie passe par le respect de l'environnement, sa protection et un développement durable. Les Accords de paix de 1996 stipulent pourtant dans leur article 67 que « les communautés indigènes et les autres [communautés] possédant des terres qui leur appartiennent historiquement et qui les ont traditionnellement administrées de manière spécifique, [verront] ce système maintenu $\gg^{36}$. Toutefois, à propos de cet article 67, les organisations « mayas $»^{37}$ estiment que les indigènes continuent d'être considérés par l'État et l'élite guatémaltèque comme des citoyens de seconde catégorie, dans la mesure où ils doivent être protégés de manière permanente par le gouvernement; elles refusent donc les politiques et les discours teintés de paternalisme. Le 22 novembre 2009, les populations indiennes de huit municipes du département de Huehuetenango ont signé la Déclaration des peuples Q'anjob'al, Chuj et Akateko libres de mines proclamant leur désapprobation de la politique nationale et leur volonté de s'unir pour la défense de leur territoire et de leur environnement.

Plusieurs leaders « mayas » ont participé aux réunions et discussions du Groupe de travail sur les populations indigènes des Nations unies (créé en 1982), notamment sur le projet de Déclaration des droits des peuples indigènes, et aux débats qui, dès 1989, ont mené à la ratification par le Guatemala de la Convention 169 de l'OIT. Cette attention internationale a renforcé les revendications des populations indiennes et leur nouvelle relation à l'État. Des contacts existent depuis longtemps entre des leaders « mayas » et d'autres dirigeants indigènes d'Amérique, notamment en vue de la tenue de sommets indigènes comme celui qui a eu lieu à Quetzaltenango en 1991, parallèlement aux grandes célébrations des 500 ans de la découverte de l'Amérique. L'année suivante, l'attribution du prix Nobel de la paix à Rigoberta Menchú

35. Au Guatemala comme dans de nombreux pays d'Amérique latine, les ressources du sous-sol sont la propriété de l'État.

36. Accords de paix 1996, art. 67.

37. Consejo de los Pueblos Mayas de Occidente, Colectivo ecologista MadreSelva, blog assembleahuehue... 
Tum, militante des droits des indigènes au Guatemala, a donné une plus grande visibilité internationale aux revendications des populations « mayas ». Toutefois, jusqu'à présent, aucune organisation n'a émergé au Guatemala pour représenter l'ensemble d'une population indigène nombreuse et, rappelons-le, très hétérogène.

L'échec du référendum de 1999 destiné à modifier la Constitution de 1985 souligne les limites des possibilités de changements et les réticences d'une partie de la société à admettre la particularité culturelle de ses populations indiennes. L'anthropologue S. Bastos a qualifié de « multiculturalisme cosmétique » les politiques des gouvernements successifs des années 2000 à l'égard des populations indigènes ${ }^{38}$. La différence culturelle au sein de la société guatémaltèque est acceptée - ou se trouve du moins en voie d'acceptation dans certains secteurs de la société, mais les facteurs économiques et sociaux expliquant l'exclusion des Indiens ne sont pas débattus publiquement, et ce bien que certains acteurs du mouvement « maya » aient exercé des responsabilités ministérielles sous le gouvernement de Alfonso Portillo (2000-2004) en tant que ministres ou secrétaires d'État à l'Éducation ou à la Culture. L'exemple du Guatemala permet également de cerner les diverses expressions politiques des stratégies d'identifications ethniques dans l'une des démocraties centraméricaines où la population indienne est majoritaire sans pour autant, comme en Bolivie ou en Équateur, contrôler l'État. L'exclusion sociale et la discrimination culturelle appliquées dans la société guatémaltèque sont héritées de son passé colonial et surtout des politiques libérales du XIX ${ }^{e}$ siècle, dont les fondements n'ont jamais été totalement remis en cause. La violence caractérise les relations entre les grands propriétaires et le reste de la population, prouvant que le conflit politique se double d'un conflit agraire. De fait, les massacres et la répression des populations civiles de la fin des années 1970 au début des années 1980 correspondent au moment où les demandes de terres des populations rurales étaient les plus fortes. Inférieurs socialement et économiquement par rapport au reste de la population «blanche » et ladina, les Indiens du Guatemala demeurent des citoyens de second rang, en raison de discriminations liées autant à leur situation sociale qu'à leur condition ethnique.

Pour de nombreux chercheurs, spécialistes de la société guatémaltèque, la candidature de Rigoberta Menchú Tum à la présidence de la République en 2007 et en 2011 a ravivé le racisme des classes possédantes et des milieux d'affaires. Par ailleurs, les politiques menées par les agences de développement et/ou onusiennes ont cristallisé les discours essentialistes, exacerbant ainsi la polarisation des discours politiques et ethniques entre « mayas »,

38. S. Bastos, « La movilización maya en Guatemala : exigiendo derechos y construyendo multiculturalidad en un contexto de postconflicto », art. cité. 
ladinos et « blancs ». Les différents groupes sociaux et ethniques composant la société guatémaltèque sont aujourd'hui conscients qu'une discrimination fondée sur la couleur de la peau, le nom de famille ou le groupe ethnique perdure depuis la signature des Accords de paix. Comme le souligne S. Bastos, le mouvement « maya » n'a ni su ni pu mettre fin à la marginalisation qui touche la population indienne. Le retour à la démocratie et la participation de certains dirigeants indiens au gouvernement n'ont pas eu pour effet de promouvoir de nouvelles politiques susceptibles d'endiguer l'un des problèmes majeurs du Guatemala : la pauvretés ${ }^{39}$.

Carine Chavarochette est anthropologue et historienne, chercheure associée au Centre de recherche et de documentation des Amériques (CREDA-CNRS) (UMR 7227). Ses thèmes de recherche sont l'anthropologie de l'eau et de l'environnement, l'anthropologie religieuse, les conflits ethniques et agraires, les frontières, le Mexique et l'Amérique centrale. Elle a publié dernièrement Frontières et identités en terres mayas MexiqueGuatemala (XIXe-XXI siècle) (Paris, L'Harmattan, 2011) et « L'eau vecteur de la compréhension des transformations sociales et religieuses d'un quartier périphérique du Sud-Est du Mexique (Chiapas) ", Journal des anthropologues (132-133, 2013, p. 291-316). cchavarochette@ymail.com 